\title{
Failure Probability of a FinFET-based SRAM cell utilizing the Most Probable Failure Point
}

\author{
Michail Noltsis ${ }^{\mathrm{a}, \mathrm{b}, *}$, Eleni Maragkoudaki ${ }^{\mathrm{a}}$, Dimitrios Rodopoulos ${ }^{\mathrm{c}}$, Francky \\ Catthoor $^{\mathrm{b}, \mathrm{c}}$, Dimitrios Soudris ${ }^{\mathrm{a}}$ \\ ${ }^{a}$ National Technical University of Athens, 9 Heroon Polytechneiou Str., 15780 Athens, \\ Greece \\ ${ }^{b}$ Katholieke University, Oude Markt 13, 3000 Leuven, Belgium \\ ${ }^{c}$ imec, Kapeldreef 75, 3001 Leuven, Belgium
}

\begin{abstract}
Application requirements along with the unceasing demand for ever-higher scale of device integration, has driven technology towards an aggressive downscaling of transistor dimensions. This development is confronted with variability challenges, mainly the growing susceptibility to time-zero and timedependent variations. To model such threats and estimate their impact on a system's operation, the reliability community has focused largely on Monte Carlo-based simulations and methodologies. When assessing yield and failure probability metrics, an essential part of the process is to accurately capture the lower tail of a distribution. Nevertheless, the incapability of widely-used Monte Carlo techniques to achieve such a task has been identified and recently, state-of-the-art methodologies focusing on a Most Probable Failure Point (MPFP) approach have been presented. However, to strictly prove the correctness of such approaches and utilize them on large scale, an examination of the concavity of the space under study is essential. To this end, we develop an MPFP methodology to estimate the failure probability of a FinFET-based SRAM cell, studying the concavity of the Static Noise Margin (SNM) while comparing the results against a Monte Carlo methodology.
\end{abstract}

Keywords: Most Probable Failure Point (MPFP), Reliability, Monte Carlo, SRAM

\footnotetext{
${ }^{*}$ Corresponding author.

Email address: mnoltsis@microlab.ntua.gr (Michail Noltsis)
} 


\section{Introduction}

Shrinking of device dimensions has brought to surface reliability issues such as aging and time-zero variability. Actually, process variation phenomena such as the random discrete dopant fluctuation (RDF) [1] and timedependent ones (like Bias Temperature Instability (BTI) and Hot Carrier Injection (HCI) [2]) deteriorate the device's performance since they significantly impact the threshold voltage among others. Aside from performance degradation and reliability threats, these phenomena cause a non-deterministic behavior of the device and therefore of the system. This stochastic nature of performance can be captured through the estimation of distributions, rather than fixed values, to describe performance metrics and device characteristics. In fact, this shift in probabilistic behavior also explains the utilization of Statistical Static Timing Analysis tools (SSTA) instead of Static Timing Analysis (STA) ones [3]. SSTA tools, first model delay from device characteristics and then propagate these distributions. Nevertheless, traditional SSTA methodology still suffers from certain inaccuracies like for example the incapability to include the spatial correlations of process variation and the delay correlation of different circuit paths [4].

To overcome these disadvantages, the reliability community has also largely focused on Monte Carlo experiments while combinations of Monte Carlo algorithms with SSTA methodology have also been discussed [5], [6]. The standard Monte Carlo approach randomly selects samples from the variation space therefore to accurately capture yield and probabilities of rare events either requires a large number of samples and prohibitive simulation times or focuses on fitting data of a reasonable number of samples to known distributions. This fitting process of the latter methodology inevitably introduces errors hence, the objective to capture failure probability values with a higher accuracy still remains. Although approaches like the Quasi-Monte Carlo technique have also been proposed, their implementation is still of questionable accuracy, especially when focusing on aggressively downscaled devices [7]. Then, the Importance Sampling technique can be used for such estimations, however, the accuracy of this approach heavily depends on the alternate distribution which ideally should be close to the final one. However, the final distribution is scarcely known [8].

The drawbacks of these conventional methods have long been identified and another approach, utilizing the Most Probable Failure Point (MPFP) has been introduced [9]. The main advantage of this technique is that it is 
distribution-independent in a sense that the metric under study (i.e. delay of a gate, Static Noise Margin of an SRAM cell e.t.c.) is not fitted to any distribution. As expected, the MPFP methodology has been since then used in many works [8], [10]. Recently, a new MPFP approach was discussed that utilizes the chi-square distribution to improve probability mass coverage and achieves even higher accuracy compared to the standard MPFP estimations [11]. Although all these works produce promising results, a fundamental aspect of this methodology has been swept under the carpet: the concavity of the metric space under study. In the case that this space is not concave, the MPFP methodology is rendered inapplicable.

Our work focuses on an implementation based on recent MPFP approaches while comparing the failure probability estimations against the typical Monte Carlo methodology. For our case-study, we focus on a FinFETbased $6 \mathrm{~T}$ SRAM cell suffering from both time-zero and time-dependent variability while examining hold stability. The novelty of our work lies on a thorough study of the concavity of the SNM space, differentiating the current paper from prior art.

The rest of this paper is organized as follows: Section 2, presents an overview of the typical MPFP methodology and discusses the recently proposed MPFP approach, focusing on its ability to produce results of higher accuracy. In Section 3 we study the concavity of SNM space. In Section 4 we compare our failure probability estimations against a typical Monte Carlo approach. After studying the SNM space, we optimize the discussed MPFP approach on Section 5. Finally, Section 6 concludes this work.

\section{Prior Art on MPFP}

Although methodologies on finding a most probable point of system failure had been utilized by mechanical and aerospace engineers, the scheme was eventually adopted by reliability engineers in electronic design and architecture [9]. Its core concept is based on the following assumptions:

- Due to process variation and aging effects, device characteristics of the circuit ( $V_{t h}$ in our case), are described by a vector $\mathbf{x}$. The space of $\mathbf{x}$ is depending on the size of the netlist, specifically the number of involved devices. For instance, for a $6 \mathrm{~T}$ SRAM cell, $\mathrm{x} \in R^{6}{ }^{1}$.

\footnotetext{
${ }^{1}$ For a memory cell, the wires in the BL/WL can easily contribute to the variability,
} 
- For every combination of $V_{t h}$ values of the involved devices, namely for every $\mathbf{x}$, corresponds a value $y$ of a metric, which in our case is the SNM. This is captured through the formulation $y(\mathbf{x})$. Of course, a mathematical formulation of the function $y(\mathbf{x})$ is extremely difficult, if not impossible, to find. Therefore, in most cases through experiments or simulation results a $y_{A}$ value is matched to a certain $\mathbf{x}_{A}$ vector.

- We assume a certain margin $Y$ for the metric value we examine. Depending on the metric under study, the failure criterion can be either $y<Y$ or $y>Y$. In our case, a sample is considered failed when $y<Y$ where $Y$ is a margin value for SNM metric. Nevertheless, assuming the metric under study is the delay of a path, it is self-evident that the failure criterion switches to $y>Y$, considering maximum delay $Y$.

- After sampling the $\mathbf{x}$ space - either exhaustively or by following an optimization method - we focus on a) identifying the most probable failure point $\mathbf{x}_{M P F P}$ (that is the point that leads to failure and is most likely to appear) and b) isolating $F$ space, that contains all the failed points and of course $\mathbf{x}_{M P F P} \in F$. Actually, locating the most probable failure point is crucial in estimating the $F$ space. Finally, the failure probability is simply $P(\mathbf{x} \in F)$.

Certain flavors of MPFP implementation can be found in the literature. Regarding the sampling of the space, as stated in [9], we can find tools that exhaustively sample the space. This provides the complete picture of $F$ and therefore the most accurate results however, leads to prohibitive simulation times. Considering that the complexity of an exhaustive search increases dramatically with the number of devices, it is crucial to examine trade-offs between a reasonable step of the sampling space. Another technique follows the assumption that the function of some devices is more significant to system's operation compared to others. Therefore, instead of sampling a space $\subset R^{6}$, one can move on a lower-dimensional space neglecting variance of other devices. For example, the work of [12] assumes a 1-D approximation while the work of [10] realizes that WL devices for an SRAM hold operation are not crucial and minimizes search space of an SRAM cell to 4-D. Other reasonably efficient sampling algorithms are also mentioned [8].

especially for large SRAM blocks. Thus $\mathbf{x}$ space can be even more complex. However, such a study is outside the scope of the current work 
Then, it is important to locate the most probable failure point that assists in estimating the failure probability. Looking into a bulk of prior art ([9], [10] and [13]) we can see that the most probable failure point is estimated from Equation 1, which represents an optimization problem. Since the MPFP point (ideally) lies on the failure boundary, the probability of this point is equal to the failure probability of the circuit under test.

$$
\max \left\{P_{\text {fail }}=\prod_{i=0}^{N-1} P\left(\left|\Delta V_{t h, i}\right| \geq x_{i}\right)\right\}
$$

Nevertheless, we have already mentioned that failure probability is $P(\mathbf{x} \in$ $F$ ) and regardless how accurate our estimations on the MPFP location are, it is important to have a solid indication of the $F$ space. This problem is discussed in [11] where the authors differentiate their implementation from previous approaches by adopting $x^{2}$ distribution and drawing hyperspherelike shapes to include all pass (or failed) samples. This methodology achieves a more accurate estimation of the $F$ space and therefore of the failure probability. Interestingly, a hypersphere sampling method is also described in [8] however, this methodology only focuses on the identification of the MPFP point and does not proceed in transforming the metric space under study.

All previous work is based on the assumption that regarding variation, failure is monotonic [9]. Specifically, it is mentioned that "as variation increases the cell will move towards failure, and when failure is reached the cell will keep failing" focusing on SRAM cells. This assumption on the concavity of the SNM space however, to the best of our knowledge, has not been proven.

The SNM of the hold operation of an SRAM cell describes how easily a cell Figure 1: Example of a butterfly curve. flips under hold conditions. In principle, SNM is extracted graphically the SNM is quantified graphically through the butterfly curve. Specifically, it is estimated as the side length of the maximum square that can be fitted inside the lobes of this curve as in Figure 1[14]. Nevertheless, stability of a cell 
can be related to other figures of merit as well. For instance, relevant work [15] develops a methodology based on a prediction tool for the estimation of read SNM, that indicates the robustness of the cell during its read operation. The effect of variability on a cell's read operation is also studied in another paper [16]. Here, we will study the concavity of the SNM hold space and then, by following the methodology described in [11], we will proceed in accurately the estimating failure probability. In general, the FinFET devices have major advantages compared to the planar CMOS technology regarding their scalability and independent gate control[17]. In our work, FinFETbased 6T SRAM cell is implemented in $10 \mathrm{~nm}$ technology nodes using MultiGate Predictive Technology Modelcards [18].

\section{Utilizing the MPFP - SNM Focus}

\subsection{Modeling Variability}

A typical 6T SRAM cell is shown in Figure 2. For our estimations, we choose to model variations on all 6 devices. This way, we achieve greater accuracy at the cost of elevated simulation times. The approach we follow is similar to the one thoroughly presented in previous work [11]. A major differentiator between the two methodologies is that we model both time-zero

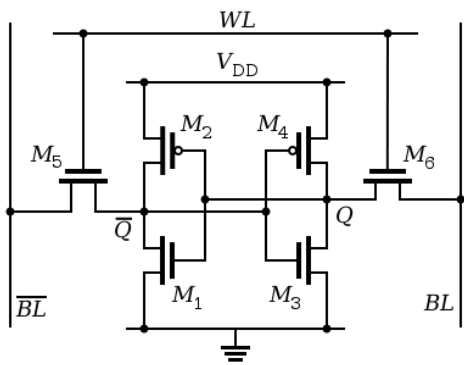

and time-dependent variability. Specifically, Figure 2: The typical 6T SRAM cell we focus on a FinFET-based, high performance SRAM cell after 3 years of continuous operation, which is a typical life-time assumption for electronics. To model variability we consider the threshold voltage of the devices distributed rather than fixed, with a Gaussian being a sufficient first-order approximation $\left(\Delta \boldsymbol{V}_{\boldsymbol{t} \boldsymbol{h}}(\boldsymbol{t}) \sim\right.$ $\left.\operatorname{Norm}\left(\left\langle\Delta V_{t h, T D}(t)\right\rangle, \sigma_{\Delta V \boldsymbol{t h}, \boldsymbol{T O T}}^{2}(t)\right)\right)$. Specifically, we assume the BTI effect to be the dominant factor of time-dependent variability and describe the mean threshold voltage shift through the formulation [19]:

$$
\left\langle\Delta V_{t h, T D}(t)\right\rangle \cong A t^{a} E_{o x}^{\gamma}
$$

Regarding the $\sigma_{V t h, T O T}$, it is depending both on time-zero and timedependent variability $\left(\sigma_{\Delta V t h, T O T}=\sigma_{\Delta V t h, 0}+\sigma_{\Delta V t h, T D}\right)[20]$. Actually, when 
time-zero variation phenomena are more aggressive, time-dependent variability is deteriorating. Specifically, for the time-dependent part, it is claimed that $[20]$

$$
\sigma_{\Delta V t h, T D}(t)=\sigma_{\Delta V t h, 0} \sqrt{\frac{<\Delta V_{t h, T D}(t)>}{100 m V}}
$$

where $\sigma_{\Delta V t h, 0}$ represents the threshold voltage shift variability at timezero due to process variation phenomena. Its value is estimated from Equation 4 where $A_{V T}$ is the Pellgrom's mismatch parameter and $W_{\text {eff }}$ is the effective width of the FinFET's channel [19], [21]

$$
\sigma_{V t h, 0}=\frac{A_{V T}}{\sqrt{2 W_{e f f} L}}
$$

and finally

$$
\sigma_{V t h, T O T}(t)=\left(\sqrt{\frac{\left\langle V_{t h, T D}(t)\right\rangle}{100 m V}}+1\right) \sigma_{V t h, 0}
$$

\subsection{Concavity of the SNM space}

Following the methodology of previous work [11], to locate the MPFP and therefore estimate the failure probability, we first need to explore the SNM space and identify the point $y(\mathbf{x})$ with the highest SNM value. To this end, using the Cadence Spectre simulator[22], we measure the SNM at each x point. It should be noted that for each device we focus on "realistic" values of threshold voltage shifts, therefore $\left|\Delta V t h_{i}\right| \leq 0.2 \mathrm{~V}$, while the step size is $50 \mathrm{mV}$. Aggressively decreasing the step size would of course lead to a more accurate view of the space, however, it would also require prohibitive simulation times. Hence, a trade-off exists between the accuracy of the analysis (in terms of space mapping) and simulation time. In fact, when studying netlists with numerous devices, inhibitive simulation times would render the exhaustive sampling of the space impractical.

After the sampling procedure, one important observation is the symmetry of the SNM space. From a closer look at Figure 2 and at basic design principles of the typical 6T SRAM cell [23], we easily realize that the cell is symmetric. This symmetry is confirmed in our analysis of the SNM space as well. Specifically, we have verified that the impact of threshold voltage shifts on any of the M1, M2 and M5 devices to the SNM value is identical 
to the same amount of shifts on the M3, M4 and M6 respectively as shown in Figures 3a-3c. Obviously, this is not the case for other combinations of devices, like for example M1 and M6 as we can verify from Figure 3d. Since a thorough representation of the whole SNM space is practically infeasible, we present certain instances where the $4 \Delta V_{t h}$ of the devices remain fixed while for the other two transistors, we sweep the threshold voltage shift and estimate the SNM.

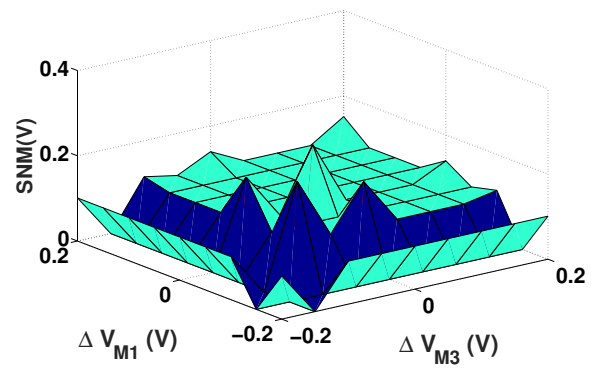

(a) SNM space for $\Delta V_{t h, M 1 / M 3}$

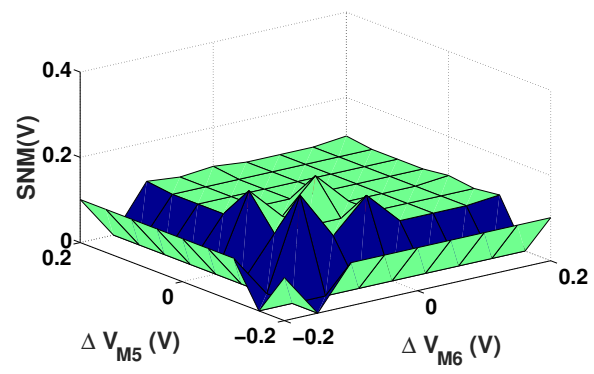

(c) SNM space for $\Delta V_{t h, M 5 / M 6}$

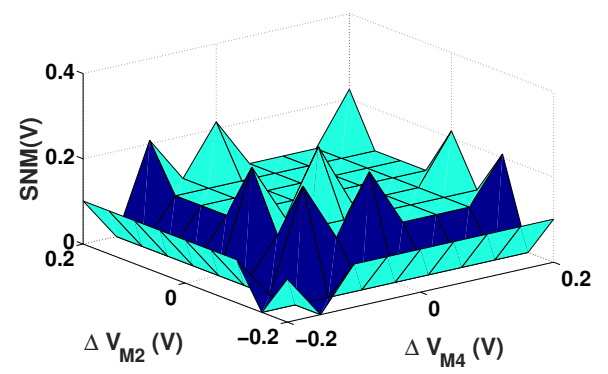

(b) SNM space for $\Delta V_{t h, M 2 / M 4}$

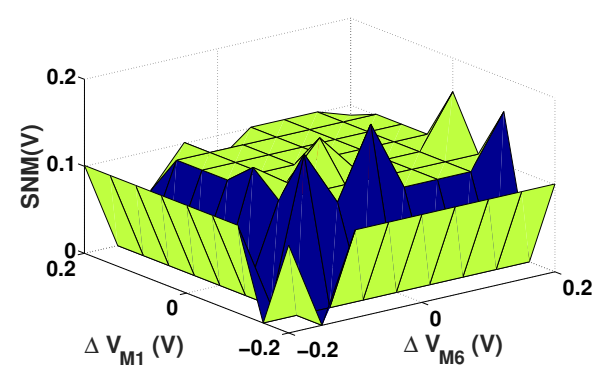

(d) SNM space for $\Delta V_{t h, M 1 / M 6}$

Figure 3: Instances of the SNM space we have observed that the SNM space contains a global maximum, while local maxima also exist. Interestingly, we have observed that this global maximum "lies in" the symmetry axis (the pairs of M1-M3, M2-M4 and M5M6 have the same threshold voltage shift). Our tool starts by initializing the threshold voltage shifts near zero while the results of the coordinate ascent 


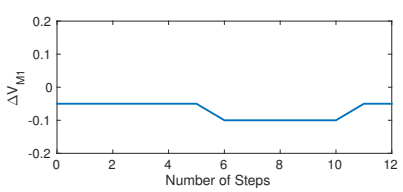

(a) $\mathrm{CA}$ for $\Delta V_{t h, M 1}$

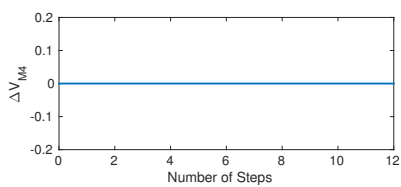

(d) CA for $\Delta V_{t h, M 4}$

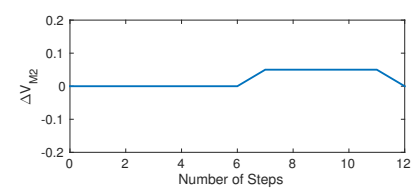

(b) CA for $\Delta V_{t h, M 2}$

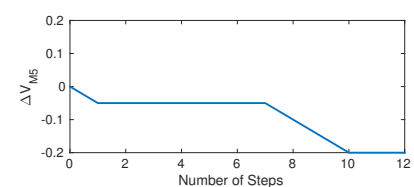

(e) $\mathrm{CA}$ for $\Delta V_{t h, M 5}$

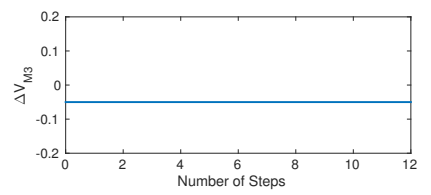

(c) CA for $\Delta V_{t h, M 3}$

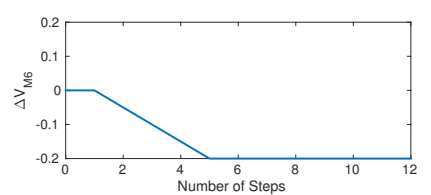

(f) CA for $\Delta V_{t h, M 6}$

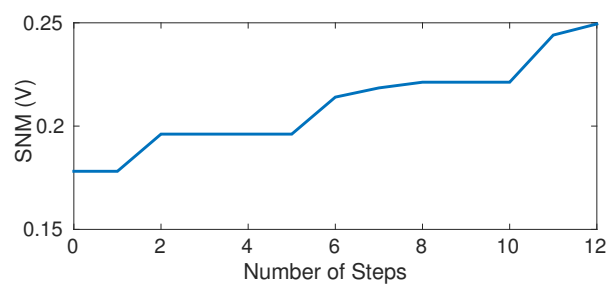

(g) CA for SNM

Figure 4: Results for the Coordinate Ascent algorithm

tool are shown in Figure 4. It is clear that as the algorithm proceeds to next steps, the SNM value increases until it reaches the maximum at the final step. We later verified through exhaustive search that this maximum is in fact the global maximum by comparing the aforementioned point with all the other sampled points of the space in terms of SNM value. It should be stressed that when utilizing a coordinate ascent algorithm and have reached a maximum, we cannot guarantee if this is the global maximum or a local one. To assess whether the first or the second case stands, a study on the concavity of the SNM space near this point is required. Should the maximum be local, we will observe points in a distance $r$ to have a SNM value higher than the aforementioned point.

A strict mathematical proof of the concavity of the space is infeasible since the exact formulation of the equation $f(\mathbf{x})=y$ is unknown. Nevertheless, we can focus on the space near the maximum and examine whether all points have an SNM value lower than the one estimated on the maximum. In addition, we expect that as distance $r$ from the maximum increases, the SNM 
value decreases. This observation would be rendered as substantial evidence on the concavity of the SNM space and show whether failure is monotonic. Figure 5 shows a representation of the SNM space near our maximum. The curves depict SNM as we are moving away from the maximum at multiple directions and each curve depicts the movement away from the maximum at a different direction. Specifically, the directions were calculated as stated in the following equation, where $\mathbf{x}_{\max }$ is the identified maximum point and $\mathbf{x}$ specifies the direction that we move parallel to each time.

$$
\mathbf{y}=t * \mathbf{x}+\mathbf{x}_{\max }
$$

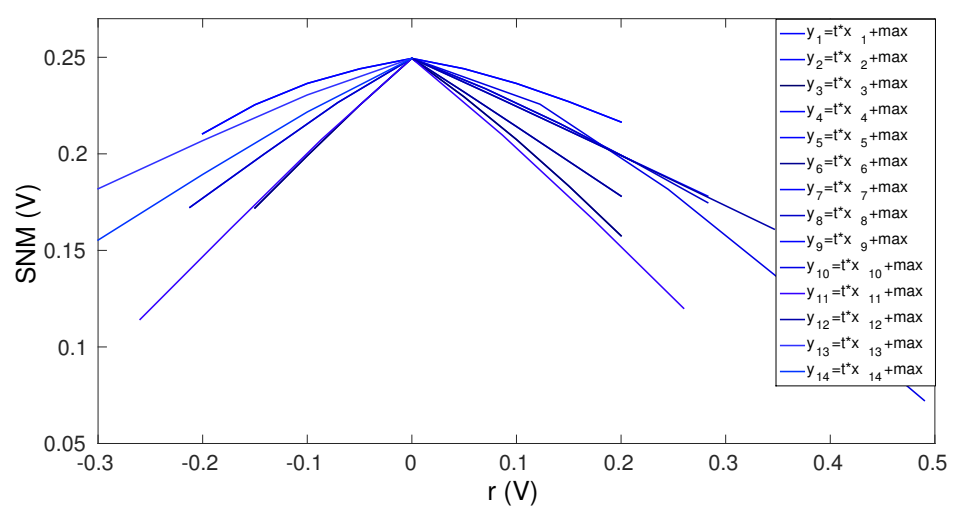

Figure 5: Concavity of the SNM space near $\mathbf{x}_{\max }$

\subsection{Estimation of the Failure Probability}

In order to estimate the failure probability, we continue by locating the $\Delta V_{t h}$ combination, namely $\mathbf{x}_{Y}$, with the minimum distance from the maximum $r_{Y}$, that leads to the failure of the cell. To achieve it we use the gradient descent algorithm[11]. According to it, we initialize the $V_{t h}$ shifts to the values of the maximum and at every step, we move towards the most 


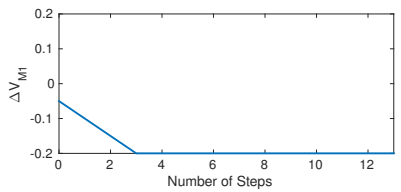

(a) GD for $\Delta V_{t h, M 1}$

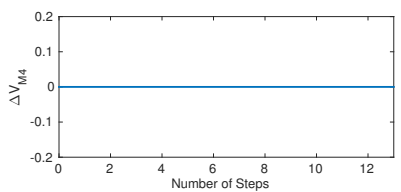

(d) GD for $\Delta V_{t h, M 4}$

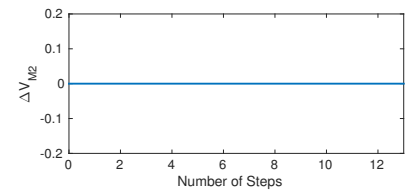

(b) GD for $\Delta V_{t h, M 2}$

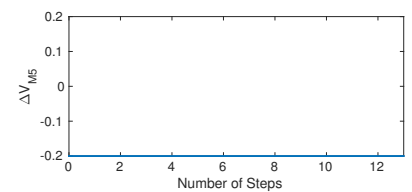

(e) GD for $\Delta V_{t h, M 5}$

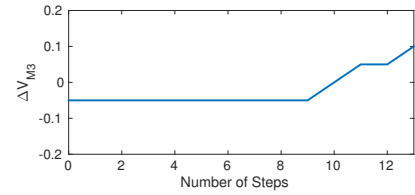

(c) GD for $\Delta V_{t h, M 3}$

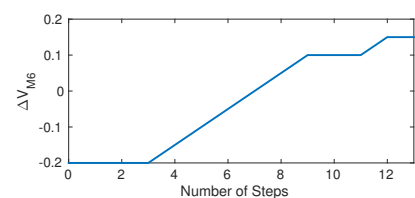

(f) GD for $\Delta V_{t h, M 6}$

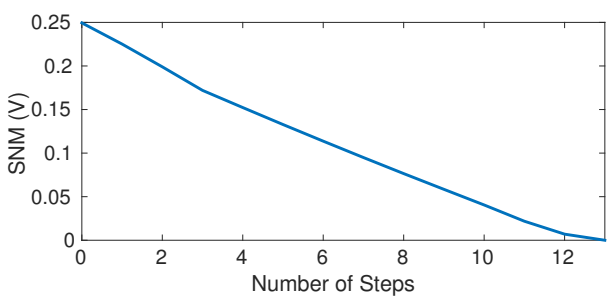

(g) GD for SNM

Figure 6: Results for the Gradient Descent algorithm

descending direction of SNM until we reach a local minimum, as shown in Figure 6. It has to be noted that this algorithm follows a different concept than the coordinate ascent. Instead of searching one coordinate at a time, it examines numerous coordinates and then moves to the point with the lower since the path that led to the maximum is not of interest.

After the identification of the most descending direction, we utilize the non central chi-square distribution $\left(\chi^{2}\right)$ to draw hypersphere-like shapes around the maximum with a radius equal to $r_{Y}$ each time. This way, we are able to estimate the failure probability at all points of the previous steps. Specifically, we will compute the probability $P(\mathbf{x} \in F)$, where the $F$ space represents the set in the $\Delta V_{t h}$ variation space that leads to samples failing the SNM criterion. Next, we use Equation 7 to calculate the random variable which is distributed according to the non central chi-squared distribution 
where $\mathrm{N}$ is the number of devices and $\sigma_{i}$ represent the standard deviations of the threshold shifts of devices. To compute the non centrality parameter $\lambda$ we use $\mu$ to represent the mean $\Delta V_{t h}$ value of each device at the global maximum.

$$
z^{2}=\sum_{i=1}^{N} \frac{x_{i}^{2}}{\sigma_{i}^{2}} \quad \lambda=\sum_{i=1}^{N} \frac{\mu_{i}^{2}}{\sigma_{i}^{2}}
$$

Finally, the failure probability is estimated using the following methodology: For each point $\mathbf{x}_{Y}$, with distance from the maximum $r_{Y}$ that the gradient descent algorithm has indicated, we calculate the failure probability regarding as $Y$ the respective SNM and using the $\chi^{2}$ distribution. Specifically, the failure probability for each design margin $Y$ satisfies Equation 8, where $C D F$ and $P D F$ are the cumulative and probability density functions of the chi-square distribution respectively.

$$
P_{\text {fail }}=1-C D F\left(r<r_{Y}\right)=1-\int_{-\infty}^{r_{Y}} P D F_{r} d r
$$

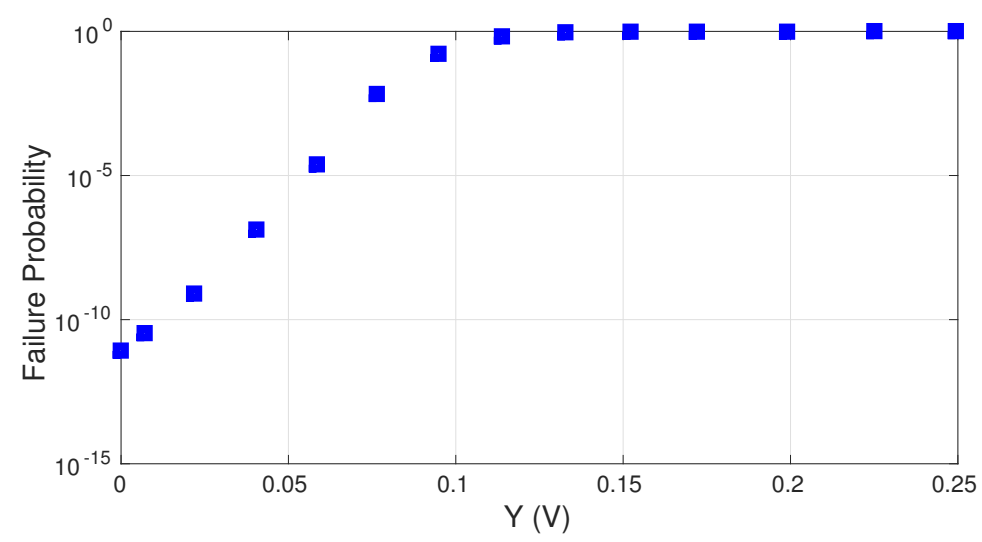

Figure 7: Failure Probability for various values of the SNM margins $Y$

The results of the failure probability versus the SNM margin $Y$ are demonstrated in Figure 7. We can see that the decrement of $Y$ leads to a lower failure probability and vice versa. This is expected, considering that failure is directly depending on the criterion and the specified SNM design margin. Hence, the decisive role of the $Y$ is obvious: when high levels of SNM margin are demanded, the failure probability of our cell rapidly drops. 


\section{Comparing MPFP to Monte Carlo}

Since the Monte Carlo (MC) technique has been largely employed to evaluate the reliability of CMOS designs, in this Section we will estimate the failure probability according to this methodology and compare it against the results of the MPFP technique we study. The simulation framework for our $\mathrm{MC}$ experiments is shown in Figure 8. First, we add time-zero variability to a median population size (namely $10^{3}$ samples) of "fresh" SRAM netlists of the same technology node, according to the mathematical formulation 4. Subsequently, we inject time-dependent variability based on Equations 2 and 5 to produce the aged netlists that suffer from both time-zero and timedependent variations. Next, through simulations, we estimate the SNM value of each sample.

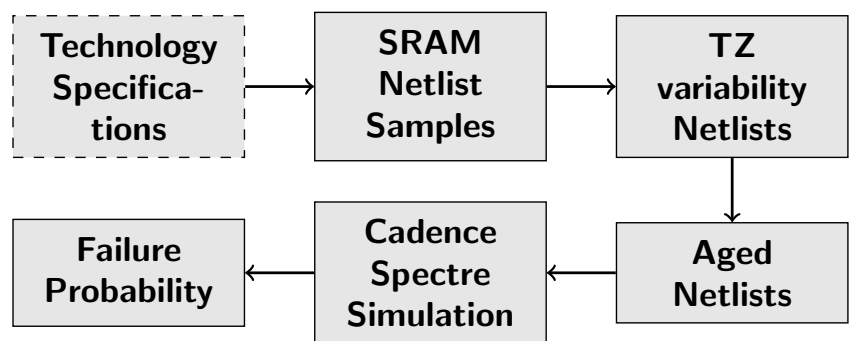

Figure 8: The Flow of our Simulation Framework

The histogram of the estimated SNMs after approximately three years of operation is presented in Figure 9a along with the design margin $Y$. Next, we present the respective failure probability estimated by the MPFP tool (Figure 9b) for the same $Y$ and we compare the two results. We can observe that for a high $Y$ value, the estimated failure probabilities by the two different methodologies are approximately equal.

For failure probabilities at significantly lower orders of magnitude (i.e. in the scale of $10^{-6}$ ) the MC methodology requires a vast population number and long simulation times. When low CPU time is a constraint, computation accuracy is poor. This is confirmed in our case, since the number of failed samples is zero when we choose a more realistic margin as shown in Figure 10. A quantitative comparison of failure probabilities for certain values of $Y$ between MC and MPFP methodologies is given in Table 1. It should be stressed that in order to alleviate prohibitive computation times, engineers work with a small population of MC samples and fit the output estimations 


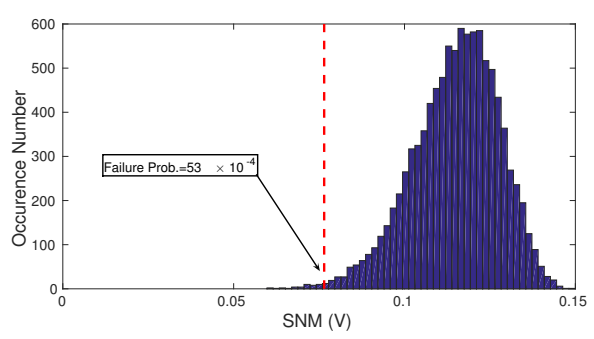

(a) Monte Carlo

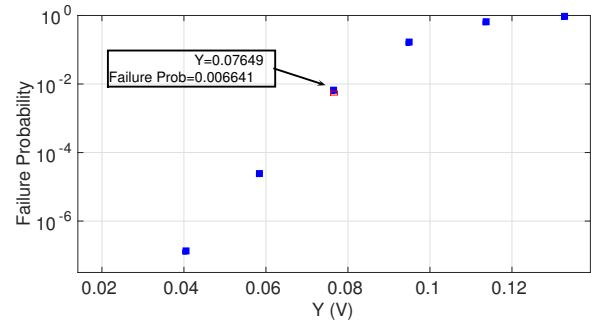

(b) MPFP

Figure 9: Comparison between MC and MPFP for a high value of $Y$

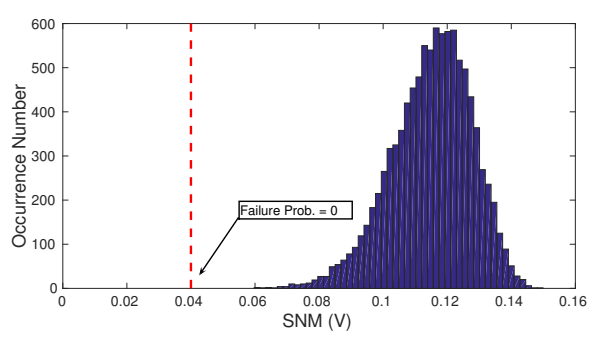

(a) Monte Carlo

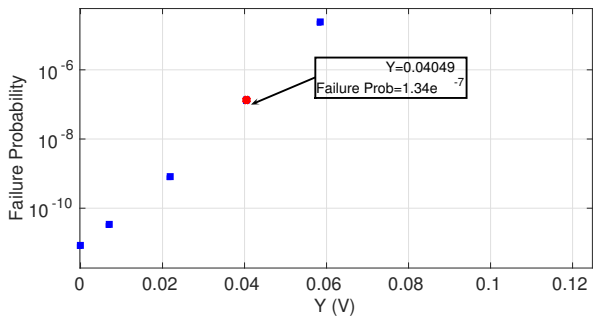

(b) MPFP

Figure 10: Comparison of Monte Carlo with MPFP for a realistic value of $Y$

to a known distribution (usually the Gaussian). However, the SNM metric is observed to have a rather prolonged tail towards lower values that the aforementioned fitting fails to capture [24]. Hence, we can conclude that while both MC and MPFP methodology are accurate for estimating failure probabilities that have high values, when focusing on probabilities of lower scales, the typical MC methodology suffers in terms of accuracy. To overcome this issue, one can select a vast number of samples however, this would render the technique inefficient.

\section{An Efficient MPFP Approach}

So far, we have shown how the MPFP approach can accurately estimate failure probability nevertheless, we have exhaustively sampled the SNM space. This required elevated simulation times. Since we have shown the concavity of the SNM space along with its symmetry, we now focus on developing new tools based on the coordinate ascent and gradient descent algorithms. This time, however, we do not sample the whole space but use Spectre "in- 
Table 1: A quantitative comparison for failure probabilities estimated by the two methodologies for certain $Y$ values.

\begin{tabular}{|l|l|l|}
\hline$Y(\mathrm{~V})$ & Failure Probability MC & Failure Probability MPFP \\
\hline 0.132 & 0.9382 & 0.9429 \\
\hline 0.113 & 0.4683 & 0.6042 \\
\hline 0.095 & 0.0656 & 0.107 \\
\hline 0.076 & 0.0055 & 0.0066 \\
\hline 0.046 & 0 & $1.34 e-7$ \\
\hline 0.020 & 0 & $8.06 e-10$ \\
\hline
\end{tabular}

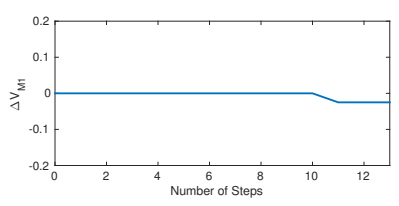

(a) Opt. CA for $\Delta V_{t h, M 1}$

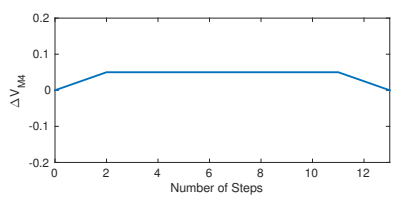

(d) Opt. CA for $\Delta V_{t h, M 4}$

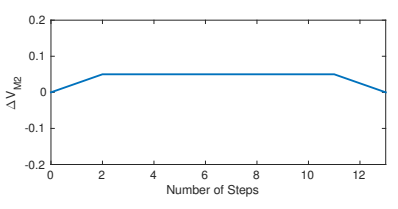

(b) Opt. CA for $\Delta V_{t h, M 2}$

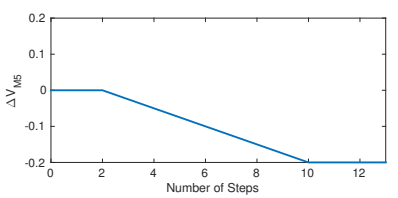

(e) Opt. CA for $\Delta V_{t h, M 5}$

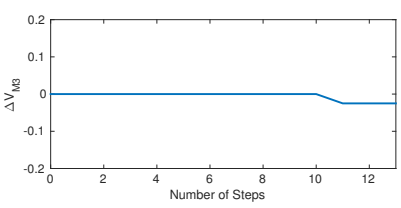

(c) Opt. CA for $\Delta V_{t h, M 3}$

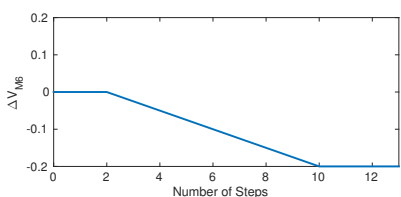

(f) Opt. CA for $\Delta V_{t h, M 6}$

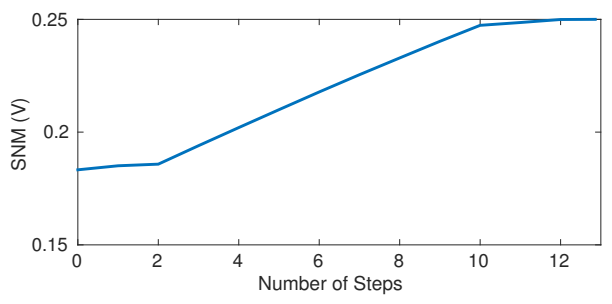

(g) Opt. CA for SNM

Figure 11: Results for the optimized Coordinate Ascent of the Efficient MPFP approach

the-loop", while the two algorithms are executed. In addition, we have improved the coordinate ascent tool exploiting the symmetry of the SNM space. We have shown and verified that the global maximum lies on the symmetry axis, therefore, our second version of the coordinate ascent tool utilizes the 


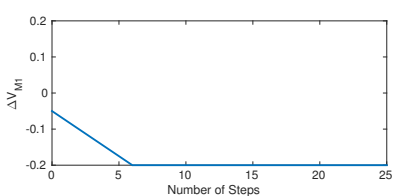

(a) GD algorithm for $\Delta V_{t h, M 1}$

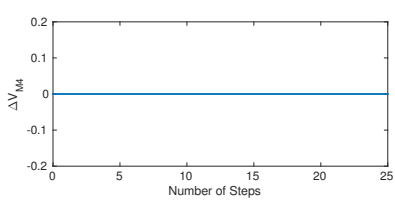

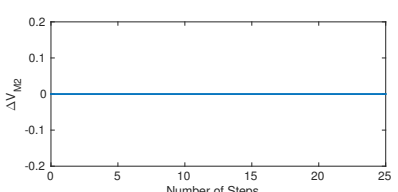

(b) GD algorithm for (c) GD algorithm for $\Delta V_{t h, M 2}$

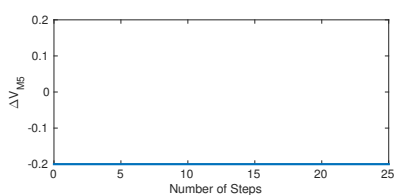

(d) GD algorithm for (e) GD algorithm for (f) GD algorithm for $\Delta V_{t h, M 4}$

$\Delta V_{t h, M 5}$

$\Delta V_{t h, M 6}$
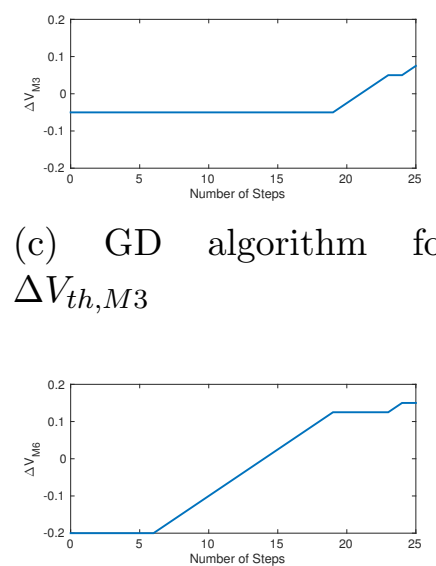

$\Delta V_{t h, M 3}$

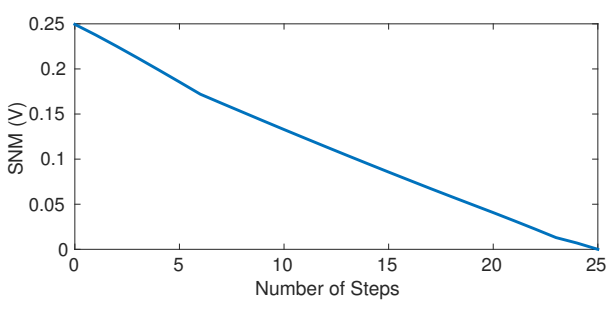

(g) GD algorithm for SNM

Figure 12: Results for the Gradient Descent algorithm of the Efficient MPFP approach

coordinates of the symmetry axis. An example of this implementation of the coordinate ascent algorithm is shown in Figure 11. Since simulation time is not a bottleneck, we have used a step size of $25 \mathrm{mV}$. Interestingly, on a typical 6T SRAM cell, the symmetry of the SNM space between devices M1M3, M2-M4 and M5-M6 will exist regardless the technology node. Hence, when studying the failure probability of a $6 \mathrm{~T}$ SRAM cell, the current efficient MPFP approach can be utilized without recurring computations on the concavity or symmetry of the SNM space.

Within the same spirit, we move forward with the gradient descent tool starting from the maximum. Because of the decreased step size, the steps to reach the minimum are nearly double. In addition, since our simulator is running "in-the-loop" with the gradient descent, the total computation time 


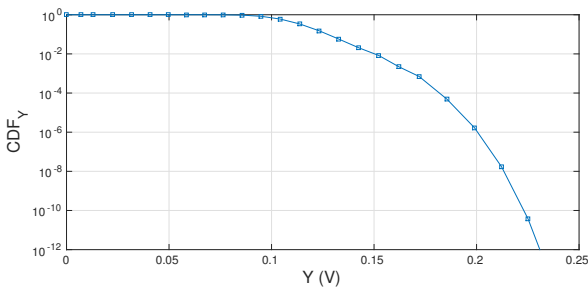

(a) $C D F_{Y}$

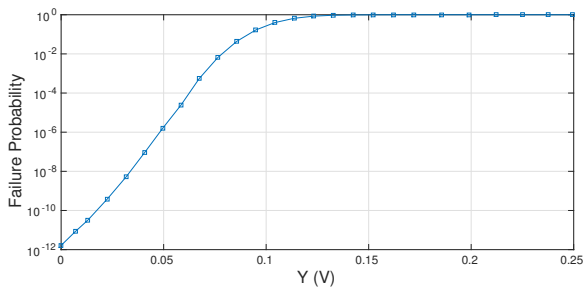

(b) $P_{\text {fail }}$

Figure 13: $C D F_{Y}$ and, eventually, the $P_{\text {fail }}$ for our SRAM cell in terms of SNM design margin

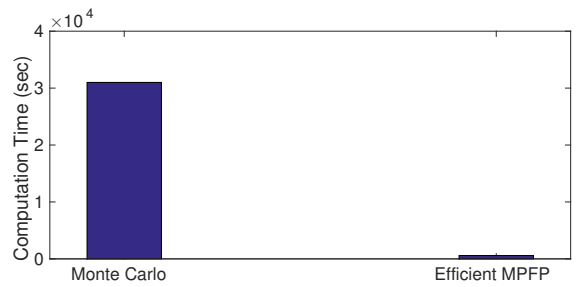

Figure 14: Difference in Computation time for the two methodologies

is negligible. In fact, we can decrease step size to achieve even higher accuracy in our estimations. The results of the optimized version of the gradient descent tool are shown in Figure 12. This time, according to Equation 8 we estimate the cumulative distribution of $Y$ and eventually the distribution of $P_{\text {fail }}$ in Figure 13. We outline the difference in computation time on a Intel CPU i3 8100 processor for our previous Monte Carlo approach versus the current efficient MPFP scheme in Figure 14.

\section{Conclusion}

In this paper we have presented a complete framework for estimating the failure probability of a 10nm FinFET-based SRAM cell suffering from process and time-dependent variations. Specifically, we focused on the hold stability and evaluated the SNM metric. We have assumed the dominant source of time-dependent variability is the BTI effect and have estimated the degradation of devices under 3 years of operation. The first step to the discussed approach was to show the concavity of the SNM space and validate the assumptions concerning the failure monotonicity of a typical 6T SRAM 
cell. We also verified the symmetry of the SNM space, a consistency of the cell's design principles. To estimate the failure probability, we exhaustively sampled the SNM space, while the core tools of our framework consist of implementing a coordinate ascent and a gradient descent algorithm. In addition, we briefly showed the advantages of the MPFP methodology compared against a simple Monte Carlo-based technique in terms of accuracy. Lastly, we moved forward by optimizing our tools to avoid exhaustive sampling of the space, since concavity has already been shown. In this new version, we exploited the symmetry of the SNM space while we "adjusted" our simulator to work on top of the coordinate ascent and gradient descent tools. Now, our final tool presents significant advantages over a typical Monte Carlo approach both in terms of accuracy and efficiency, saving up to $97 \%$ in terms of computation time.

\section{References}

[1] K. Takeuchi, T. Fukai, T. Tsunomura, A. T. Putra, A. Nishida, S. Kamohara, T. Hiramoto, Understanding random threshold voltage fluctuation by comparing multiple fabs and technologies, in: IEEE International Electron Devices Meeting (IEDM), pp. 467-470.

[2] I. Lahbib, M. A. Doukkali, P. Martin, G. Imbert, Hot carrier injection and negative bias temperature instability induced nmos and pmos degradation on cmos ring oscillator, in: Reliability and Maintainability Symposium (RAMS).

[3] C. Forzan, D. Pandini, Why we need statistical static timing analysis, in: IEEE 25th International Conference on Computer Design, pp. 91-96.

[4] R. Garg, S. P. Khatri, Analysis and Design of Resilient VLSI Circuits, Springer, 2010.

[5] V. Veetil, Y.-H. Chang, D. Sylvester, D. Blaauw, Efficient smart monte carlo based ssta on graphics processing units with improved resource utilization, in: ACM/IEEE 47th Design Automation Conference (DAC), pp. 793-798.

[6] J. Cong, K. Gururaj, W. Jiang, B. Liu, K. Minkovich, B. Yuan, Y. Zou, Accelerating monte carlo based ssta using fpga, in: ACM/SIGDA 18th, 
Inter. symposium on Field programmable gate arrays (FPGA), pp. 111114 .

[7] M. Noltsis, P. Weckx, D. Rodopoulos, F. Cathoor, D. Soudris, Accuracy of quasi-monte carlo technique in failure probability estimations, in: IEEE, Inter. Conference on IC Design and Technology (ICICDT).

[8] M. Rana, R. Canal, Ssfb: A highly-efficient and scalable simulation reduction technique for sram yield analysis, in: Design, Automation and Test in Europe Conference and Exhibition (DATE).

[9] D. Khalil, M. Khellah, N.-S. Kim, Y. Ismail, T. Karnik, V. De, Sram dynamic stability estimation using mpfp and its applications, Microelectronics Journal, Elsevier 40 (2009) 1523-1530.

[10] D. Rodopoulos, Y. Sazeides, F. Catthoor, C. Nicopoulos, D. Soudris, Sensitivity of sram cell most probable snm failure point to timedependent variability, in: SELSE Silicon Errors in Logic System Effects.

[11] D. Rodopoulos, Y. Sazeides, F. Catthoor, C. Nicopoulos, D. Soudris, Approximating standard cell delay distributions using the most probable failure point, in: Workshop on Early Reliability Modeling for Aging and Variability in Silicon Systems.

[12] M. Khellah, Y. Ye, N. S. Kim, D. Somasekhar, G. Pandya, K. Z. Ali Farhang, C. Webb, V. De, Wordline \& bitline pulsing schemes for improving sram cell stability in low-vcc 65nm cmos designs, in: Symposium on VLSI Circuits.

[13] S. Ganapathy, R. Canal, D. Alexandrescu, E. Costenaro, A. Gonzalez, A. Rubio, Informer: An integrated framework for early-stage memory robustness analysis, in: Design, Automation and Test in Europe Conference and Exhibition (DATE).

[14] E. Seevinck, F. List, J. Lohstroh, Static-noise margin analysis of mos sram cells, IEEE Journal of Solid-State Circuits 22 (1987) 748-758.

[15] J. L. Merino, S. A. Bota, R. Picos, J. Segura, Alternate characterization technique for static random-access memory static noise margin determination, International Journal of Circuit Theory and Applications 41 (2012). 
[16] K. Takeuchi, T. Mizutani, T. Saraya, H. Shinohara, M. Kobayashi, T. Hiramoto, Correlation between static random access memory powerup state and transistor variation, Japanese Journal of Applied Physics 56 (2017).

[17] A. Carlson, Z. Guo, S. Balasubramanian, R. Zlatanovici, T.-J. K. Liu, B. Nikolic, Sram readwrite margin enhancements using finfets, IEEE Transactions on Very Large Scale Integration (VLSI) Systems 18 (2010).

[18] A. S. University, Predictive technology model, http://ptm.asu.edu/, 2012. [Online].

[19] H. K. et al., Scaling of bti reliability in presence of time-zero variability, in: 2014 IEEE International Reliability Physics Symposium.

[20] P. Weckx, B. Kaczer, M. Toledano-Luque, P. Raghavan, J. Franco, P. J. Roussel, G. Groeseneken, F. Catthoor, Implications of bti-induced timedependent statistics on yield estimation of digital circuits, IEEE Transactions on Electron Devices 61 (2014) 666-673.

[21] K. J. Kuhn, M. D. Giles, D. Becher, P. Kolar, A. Kornfeld, R. Kotlyar, S. T. Ma, A. Maheshwari, S. Mudanai, Process technology variation, IEEE Transactions on Electron Devices 58 (2011) 2197-2208.

[22] Cadence, Spectre circuit simulator, https://www.cadence.com, 2004. [Online].

[23] W. Neil, H. David, CMOS VLSI Design: A Circuits and Systems Perspective, Addison-Wesley, 2011.

[24] X. Wang, B. Cheng, J. B. Kuang, Statistical variability and reliability and the impact on corresponding 6t-sram cell design for a 14-nm node soi finfet technology, IEEE Design \& Test 30 (2013) 18-28. 